\title{
Relationship between Alanine and Aspartate Transaminases (ALT and AST) and Fatty Liver on Ultrasound
}

\author{
ADEENA KHAN ${ }^{1}$, SADIA ALI ${ }^{2}$, MUHAMMAD USMAN UL HAQ ${ }^{3}$, MAMOONA SULTAN ${ }^{4}$, SABA MAQSOOD $^{5}$, SANA \\ AKHTAR 6 \\ ${ }^{1,4}$ Assistant Consultant, King Saud University Medical City, Riyadh Saudi Arabia \\ ${ }^{2,5}$ Assistant Professor Radiology, Avicenna Hospital and Medical College \\ ${ }^{3}$ Jarir Medical Centre, Riyadh, Saudi Arabia \\ ${ }^{6}$ Assistant Consultant Radiologist, Islamabad Diagnostic Center. Evercare Lahore \\ Correspondence to Dr. Adeena Khan, Email: khan.adeena@yahoo.com Cell: 00966599720990
}

\begin{abstract}
Background: Non-alcoholic Fatty liver disease, also known as NAFLD is said to result from a number of disorders such as insulin resistance, Obesity \& diabetes mellitus possibly as a result of an unhealthy and sedentary life style. The prevalence of NAFLD is stated to be $25.23 \%$, but it ranges between 115 to $45 \%$ in Asian countries globally. Plasma aminotransferases (aspartate1aminotransferase [AST] \& alanine aminotransferase [ALT]) are increased in patients with non-alcoholic fatty liver disease (NAFLD).

Aim:To find the relationship between alanine and aspartate transaminases (ALT and AST) and fatty liver on ultrasound.

Study design \& duration: Retrospective Study, September 2017-2018.

Settings: Radiology Department of Avicenna Medical College and Hospital, Lahore.

Methods: 274 patients present with age of 18 years and above with demographic features as body mass index, grade of fatty liver and also noted ALT, AST and size of fatty liver were included from the study. Grade I was labeled if there was increased hepatic echogenicity but visible periportal \& diaphragmatic echogenicity. Grade II was considered if increased hepatic echogenicity causes imperceptible periportal echogenicity, without obscuration of diaphragm. Grade III was considered as marked increase in liver echogenicity with imperceptible periportal echogenicity and diaphragm. The patients were then followed for the Alanine transaminase (ALT) and Aspartate transaminase (AST)

Results: In this study The mean age of patients $53.8 \pm 11.94$. There were $116(42.3 \%)$ male and $158(57.6 \%)$ females. Majority of patients were of grade II fatty liver 126(45.9\%). Grade III fatty liver was found in $110(40.1 \%)$ and grade I fatty liver was found in 38(13.8\%). hepatomegaly was seen in $153(55.8 \%)$ patients. None of the grade I fatty liver patients were found to have raised ALT or AST. Raised ALT was found in 21 patient of grade II and 15 of grade III fatty liver patients. Raised AST was found in 20 of grade II and 7 had grade III fatty liver patients.

Conclusion: The conclusion of this study that mean of ALT increased as compare to AST. None of the grade I fatty liver patients were found to have raised ALT or AST. Raised ALT was found in 21 patient of grade II and 15 of grade III fatty liver patients.

Keywords: Fatty liver disease, Grade I, II, III, AST, ALT
\end{abstract}

\section{INTRODUCTION}

Non-alcoholic Fatty liver1disease, also known as NAFLD is thought to be caused by a number of disorders such as insulin resistance, obesity \& diabetes mellitus possibly as a result of an un-healthy \& sedentary life style ${ }^{1,2}$.Normally NAFLD is not, considered a serious illness, if remains untreated or un-diagnosed, it can lead to hepatic complications \& liver cirrhosis. Though it was the most serious health problem in western countries ${ }^{3}$. NAFLD, is now being given full consideration in Asia, due to increase in obesity \& related with metabolic health complications ${ }^{4}$. The prevalence of NAFLD is stated to be $25.23 \%$, while it ranges between 15 to $45 \%$ in Asian countries globally ${ }^{3}$.

Normally, plasma aminotransferases (aspartate aminotransferase $[\mathrm{AST}] \&$ alanine aminotransferase [ALT]) are increased in nonalcoholic fatty liver disease (NAFLD)patients. But, the factors behind their elevation remain unknown. Previous research has suggested that

Received on 16-02-2021

Accepted on 18-06-2021 patients with normal level of plasma aminotransferase may be at risk of developing NASH \& fibrosis, but the findings have been mixed, and the issues related with NASH in this setting are unknown ${ }^{5,6}$.

Generally, population visits gastroenterologists when they find high levels of serum alanine transaminases. That is the main reason in many studies it is found that NAFLD diagnosis is made on the levels of aspartame transaminase (AST) and alanine transaminase (ALT) $)^{7,8}$. In clinical settings blood lipid profile and serum AST, ALT levels are done along with impaired blood sugar fasting levels ${ }^{9,10}$. All such biomarkers have a significant role in the development of NAFLD. These biochemical markers help in understanding the pathogenesis and future outcome of the disease so that early intervention can be done to reduce the risk of cirrhosis, hepatocellular carcinoma ${ }^{11}$.

\section{METHODOLOGY}

This retrospective study was conducted from September 2017 to 2018 in the Department of Radiology, Avicenna Medical College and Hospital, Lahore after approval from 
the Ethical Committee. Written consent was obtained from patients. 274 sample size with $80 \%$ power of test and $5 \%$ level of significance by taking expecting percentage of NAFLD. Patients present with age of 18 years and above with demographic features as body mass index, grade of fatty liver and also noted ALT, AST and size of fatty liver were included from the study. Patients with Heart failure, Hepatitis A,B or C, Wilson disease, hemochromatosis, drinking alcohol, taking hepatotoxic medications or any liver related disease were excluded from the study.

Grade I was labeled if there was improved hepatic echogenicity but visible periportal \& diaphragmatic echogenicity.Grade II was considered if increased hepatic echogenicity causes imperceptible periportal echogenicity, without obscuration of diaphragm. Grade III was considered as marked increase in liver echogenicity with imperceptible periportal echogenicity and diaphragm. The patients were then followed for the Alanine transaminase (ALT) and Aspartate transaminase (AST) and their values were recorded.

Data was entered in SPSS 21. Age, BMI, liver size, Alanine transaminase and Aspartate transaminase levels were presented ad mean and standard deviation. Categorical data like gender \& grade of fatty liver were presented as frequencies \& percentage.

\section{RESULTS}

Total 274 patients with fatty liver were included in the study. The mean age was $53.8 \pm 11.94$. There were $116(42.3 \%)$ male and $158(57.6 \%)$ females with male to female ratio of 1.5:2 (Table 1). Out of 274 patients, hepatomegaly was seen in $153(55.8 \%)$ patients. Table: 1 Out of total patients having hepatomegaly, 12(8.4\%) had grade I fatty liver, 66(43.2\%) had grade II \& $75(49 \%)$ had grade III fatty liver (Figure 1).

The mean BMI was $32.5 \pm 5.90$. The raised BMl was found $254(93 \%)$ with $p$ value $<0.05$. It was found to be higher in $93(59.2 \%)$ of females having average BMI of $34.6 \pm 6.3$ as compared to $47(40.7 \%)$ of males with average BMI of $29.5 \pm 3.9$ ( $p$ value $=0.20$ ) Table: $268(24.8 \%$ ) patients had grade I disease, $142(51.8 \%)$ had grade II disease and $64(23.4 \%)$ had grade II disease. The mean of grade I, II \& II as shown in table 3'. 36(13.13\%) were found to have raised ALT and 27(9.85\%) were found to have raised AST. ALT value range was between 14.4-163.8 IU/L with mean of $34.3 \mathrm{IU} / \mathrm{L} \pm 24.1$, while $A S T$ value was in range between 12.5-98.2 IU/L having mean of 22.8IU/L \pm 13.5 (Table 4). None of the grade I fatty liver patients were found to have raised ALT or AST. Raised ALT was found in 21 patients of grade II and 15 of grade III fatty liver patients. Raised AST was found in 20 of grade II and 7 had grade III fatty liver patients.

Table1:Distribution of Gender \& Grades of Fatty Liver

\begin{tabular}{|l|l|l|}
\hline \multirow{2}{*}{ Gender } & & Frequency (\%) \\
\hline \multirow{2}{*}{ Hepatomegaly } & Male & $116(42.3 \%)$ \\
\cline { 2 - 3 } & Female & $158(57.6 \%)$ \\
\cline { 2 - 3 } & Yes & $153(55.83 \%)$ \\
\hline
\end{tabular}

Table 2: Gender distribution of raised BMI
\begin{tabular}{|l|l|l|}
\hline & Raised BMI & Mean \pm SD \\
\hline Yes & $254(93 \%)$ & $32.5 \pm 5.9$ \\
\hline No & $20(7 \%)$ & \\
\hline Female & $93(59.2 \%)$ & $34.6 \pm 6.3$ \\
\hline Male & $47(40.7 \%)$ & $29.5 \pm 3.9$ \\
\hline
\end{tabular}

Table 3: Distribution of grades of disease
\begin{tabular}{|l|l|l|l|}
\hline & Grade I & Grade II & Grade III \\
\hline Yes & $68(24.8 \%)$ & $142(51.8 \%)$ & $64(23.4 \%)$ \\
\hline Enlarged Liver & $10(13.6 \%)$ & $73(26.6 \%)$ & $52(19 \%)$ \\
\hline Mean__SD & $20.12 \pm 9.73$ & $23.57 \pm 15.98$ & $23.72 \pm 14.07$ \\
\hline
\end{tabular}

Table 4: Mean value of Raised ALT \& AST
\begin{tabular}{|l|l|l|l|}
\hline & Raised ALT & Raised AST & Pvalue \\
\hline Mean \pm SD & $22.74 \pm 14.25$ & $31.92 \pm 19.93$ & 0.88 \\
\hline Male & $22.89 \pm 13.85$ & $32.82 \pm 22.91$ & 0.52 \\
\hline Female & $22.65 \pm 13.58$ & $31.2 \pm 16.4$ & \\
\hline Mean value IU/ml & $88 \pm 26.7$ & $57.5 \pm 17.8$ & \\
\hline Value range IU/ml & $63-163.8$ & $43-98.2$ & \\
\hline
\end{tabular}

Figure 1: Hepatomegaly $(n=153)$

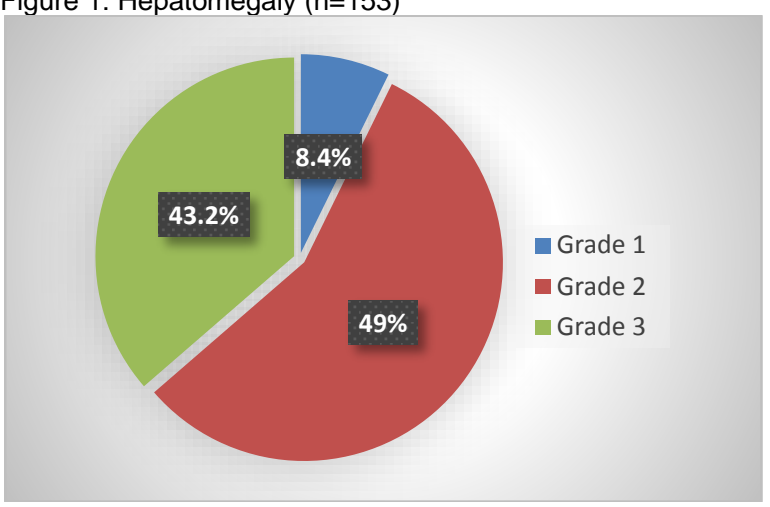

Figure 2: Raised Transaminase levels

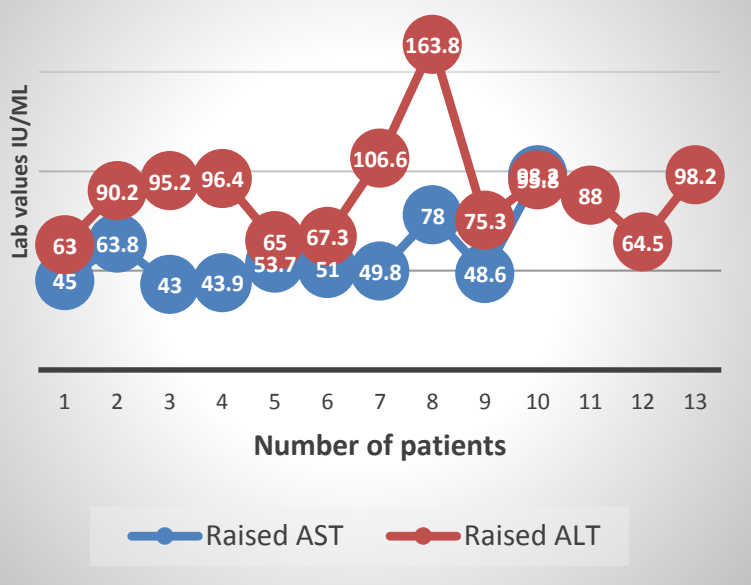

\section{DISCUSSION}

Nonalcoholic fatty liver disease and its subtype, nonalcoholic steatohepatitis, affect approximately $30 \%$ and $5 \%$ of the US population, respectively. In nonalcoholic steato-hepatitis patients, half deaths are due to malignancy \& cardiovascular disease, however awareness of this issue remains low ${ }^{12}$. 
Growing morbidity of non-alcoholic fatty liver and its association with steatohepatitis, cirrhosis, hepatocellular carcinoma, cardiovascular disease and diabetes mellitus are compelling enough to found its correlation with hepatocytes predominant amninotransferases (ALT/AST), which are the primary investigations prescribed by the clinician after the diagnosis of fatty liver.

We found ALT/AST the poor indicators of hepatic steatosis which were raised AST \& ALT only in $13.13 \%$ and $9.85 \%$ respectively. All patients with raised ALT/AST were found to be of grade II and III fatty liver with no significant difference in the number of patients between these grades. None of the grade I fatty liver patients were found to have raised ALT/AST values. According to the American College of Gastroenterology (ACG) Clinical Guidelines 2017, many patients of NAFLD may have normal liver chemistry and unlike alcoholic fatty liver, nonalcoholic fatty liver disease shows no peculiar pattern of increase in ALT or AST although ALT levels are found to be higher than AST levels but rarely above $300 I U / L^{13}$. Another study by Musso G, et al. in 2011 labeled ALT and AST as not a useful predictor of NAFLD ${ }^{14}$.Liver enzymes are neither sensitive nor specific for diagnosis of NAFLD ${ }^{15,16}$.Similar results were seen in our fatty liver patients having more raised ALT than AST with ranges between 14.4-163.8 IU/L and12.5-98.2 IU/L respectively. Contrary to this in one study by Sanyal, et al. in 2015 conducted on diabetic patients, non-alcoholic fatty liver is significantly correlated with higher AST/ALT and found to be raised in $31 \%$ of patients with positive predictive value of around $91.4 \%$ suggesting it to be a good detector of NAFLD ${ }^{17}$.

In many other studies, raised BMI (93\%) in our study was also found to be associated with disease of fatty liver and no significant difference in male and females BMI. Benedict $\mathrm{M}$, et al. found raised $\mathrm{BMI}$ in $80 \%$ of the patients ${ }^{18}$.Some studies labelled obesity as a biggest risk factor of fatty liver like many other studies ${ }^{19}$.

One more study done in 2009 , female predominance was seen in our fatty liver patients but variable trend is noticed in other studies ${ }^{20}$. Male predominance was seen in one study, while no gender predominance in other ${ }^{21}$. Our raised AST/ALT were found to be more in males. Male and old age are significantly associated with higher risk of developing NAFLD ${ }^{19,22}$.

Mean age of our patients was around 53.8 years similar to previous study showing average age of 57 years ${ }^{23}$. Majority $(51.2 \%)$ of our patients were of grade II followed by grade III (23.4\%) but Hepatomegaly was found to be more in grade III (52.7\% of patients) than grade II (40\%) hitch might be related to be increased fatty content in hepatocytes. Although histological grading is not synonymous with sonographic grading but in one study moderate histological grading was seen in most of the patients $(61.3 \%)$ than severe ${ }^{15}$.

The limitations of the study were sample size and fatty liver evaluation by ultrasound. We need large scale of prospective study to validate our observation. Ultrasound is the routine investigation done in fatty liver patients and is considered cost effective. Although histological analysis is gold standard and no imaging modality can reliably confirm diagnosis, stage the disease and determine the justification for the need of therapy. But still we did not perform liver biopsy because of its invasiveness, potential complications and cost.

\section{CONCLUSION}

The conclusion of this study that mean of ALT increased as compare to AST. None of the grade I fatty liver patients were found to have raised ALT or AST. Raised ALT was found in 21 patient of grade II and 15 of grade III fatty liver patients. Raised AST was found in 20 of grade II and 7 had grade III, fatty liver patients.

Conflict of interest: Nil

\section{REFERENCES}

1. Hannah WN, Harrison SA. Lifestyle and dietary interventions in the management of nonalcoholic fatty liver disease. Digestive diseases and sciences. 2016;61(5):1365-74.

2. Arendt BM, Comelli EM, Ma DW, Lou W, Teterina A, Kim T, et al. Altered hepatic gene expression in nonalcoholic fatty liver disease is associated with lower hepatic $n-3$ and $n-6$ polyunsaturated fatty acids. Hepatology. 2015;61(5):1565-78.

3. Younossi ZM, Koenig AB, Abdelatif D, Fazel Y, Henry L, Wymer M. Global epidemiology of nonalcoholic fatty liver disease-meta-analytic assessment of prevalence, incidence, and outcomes. Hepatology. 2016;64(1):73-84.

4. Ashtari S, Pourhoseingholi MA, Zali MR. Non-alcohol fatty liver disease in Asia: Prevention and planning. World journal of hepatology. 2015;7(13):1788.

5. Francque SM, van der Graaff D, Kwanten WJ. Non-alcoholic fatty liver disease and cardiovascular risk: pathophysiological mechanisms and implications. Journal of hepatology. 2016;65(2):425-43.

6. Chalasani N, Younossi Z, Lavine JE, Charlton M, Cusi K, Rinella $M$, et al. The diagnosis and management of nonalcoholic fatty liver disease: practice guidance from the American Association for the Study of Liver Diseases. Hepatology. 2018;67(1):328-57.

7. Wai JW, Fu C, Wong VW-S. Confounding factors of noninvasive tests for nonalcoholic fatty liver disease. Journal of Gastroenterology. 2020;55:731-41.

8. Newsome PN, Cramb R, Davison SM, Dillon JF, Foulerton M, Godfrey EM, et al. Guidelines on the management of abnormal liver blood tests. Gut. 2018;67(1):6-19.

9. Swain M, Nath P, Parida PK, Narayan J, Padhi PK, Pati GK, et al. Biochemical profile of nonalcoholic fatty liver disease patients in eastern India with histopathological correlation. Indian Journal of Clinical Biochemistry. 2017;32(3):306-14.

10. Li P-J, Jin T, Luo D-H, Shen T, Mai D-M, Hu W-H, et al. Effect of prolonged radiotherapy treatment time on survival outcomes after intensity-modulated radiation therapy in nasopharyngeal carcinoma. Plos one. 2015;10(10):e0141332.

11. Alterio A, Alisi A, Liccardo D, Nobili V. Non-alcoholic fatty liver and metabolic syndrome in children: a vicious circle. Hormone research in paediatrics. 2014;82(5):283-9.

12. Rinella ME. Nonalcoholic fatty liver disease: a systematic review. Jama. 2015;313(22):2263-73.

13. Kwo PY, Cohen SM, Lim JK. ACG clinical guideline: evaluation of abnormal liver chemistries. Official journal of the American College of Gastroenterology| ACG. 2017;112(1):1835.

14. Byrne CD, Targher G. NAFLD: a multisystem disease. Journal of hepatology. 2015;62(1):S47-S64.

15. Popescu M, Popescu I, Stanciu M, Cazacu S-M, Ianoşi N-G, Comănescu MV, et al. Non-alcoholic fatty liver diseaseclinical and histopathological aspects. Rom J Morphol Embryol. 2016;57(4):1295-302. 
16. Rau M, Schilling A-K, Meertens J, Hering I, Weiss J, Jurowich $\mathrm{C}$, et al. Progression from nonalcoholic fatty liver to nonalcoholic steatohepatitis is marked by a higher frequency of Th17 cells in the liver and an increased Th17/resting regulatory $\mathrm{T}$ cell ratio in peripheral blood and in the liver. The Journal of Immunology. 2016;196(1):97-105.

17. Sanyal D, Mukherjee P, Raychaudhuri M, Ghosh S, Mukherjee S, Chowdhury S. Profile of liver enzymes in nonalcoholic fatty liver disease in patients with impaired glucose tolerance and newly detecteduntreated type 2 diabetes. Indian journal of endocrinology and metabolism. 2015;19(5):597.

18. Benedict $M$, Zhang $X$. Non-alcoholic fatty liver disease: An expanded review. World journal of hepatology. 2017;9(16):715.

19. Pappachan JM, Babu S, Krishnan B, Ravindran NC. Nonalcoholic fatty liver disease: a clinical update. Journal of Clinical and Translational Hepatology. 2017;5(4):384.
20. Farrell GC, Larter CZ. Nonalcoholic fatty liver disease: from steatosis to cirrhosis. Hepatology. 2006;43(S1):S99-S112.

21. Tsiplakidou M, Tsipouras MG, Giannakeas N, Tzallas AT, Manousou P. Automated detection of liver Histopathological findings based on biopsy image processing. Information. 2017;8(1):36.

22. Singh S, Kuftinec GN, Sarkar S. Non-alcoholic fatty liver disease in South Asians: a review of the literature. Journal of clinical and translational hepatology. 2017;5(1):76.

23. Puchner SB, Lu MT, Mayrhofer T, Liu T, Pursnani A, Ghoshhajra BB, et al. High-risk coronary plaque atcoronary CT angiography is associated with nonalcoholic fatty liver disease, independent of coronary plaque and stenosis burden: results from the $R$

24. OMICAT II trial. Radiology. 2015;274(3):693-701. 